\title{
AROUND EFIMOV'S DIFFERENTIAL TEST FOR HOMEOMORPHISM
}

\author{
VICTOR ALEXANDROV
}

\begin{abstract}
In 1968, N. V. Efimov proved the following remarkable theorem:
Let $f: \mathbb{R}^{2} \rightarrow \mathbb{R}^{2} \in C^{1}$ be such that $\operatorname{det} f^{\prime}(x)<0$ for all $x \in \mathbb{R}^{2}$ and let there exist a function $a(x)>0$ and constants $C_{1} \geqslant 0, C_{2} \geqslant 0$ such that the inequalities $|1 / a(x)-1 / a(y)| \leqslant C_{1}|x-y|+C_{2}$ and $\left|\operatorname{det} f^{\prime}(x)\right| \geqslant a(x)|\operatorname{curl} f(x)|+a^{2}(x)$ hold true for all $x, y \in \mathbb{R}^{2}$. Then $f\left(\mathbb{R}^{2}\right)$ is a convex domain and $f$ maps $\mathbb{R}^{2}$ onto $f\left(\mathbb{R}^{2}\right)$ homeomorphically.

Here curl $f(x)$ stands for the curl of $f$ at $x \in \mathbb{R}^{2}$.

This article is an overview of analogues of this theorem, its generalizations and applications in the theory of surfaces, theory of global inverse functions, as well as in the study of the Jacobian Conjecture and the global asymptotic stability of dynamical systems.

Keywords: Efimov's theorem, Euclidean 3-space, immersed surface, Riemannian metric, Gauss curvature, Milnor's Conjecture, diffeomorphism, Jacobian Conjecture, global asymptotic stability of a dynamical system.

Mathematics subject classification (2010): 53C42, 53C40, 53A05, 26B10, 14R15, 34D23, 37C75, 58C15.
\end{abstract}

\section{INTRODUCTION}

One of the greatest achievements of 19th-century mathematics was the creation of Lobachevsky geometry, or, as it is now customary to say, hyperbolic geometry. At that time, the issue of internal consistency of such geometry was of fundamental importance. The first step in this direction was made by Eugenio Beltrami in 1868 in his article "Experience in the interpretation of non-Euclidean geometry", see [11. He proved that special domains of the Lobachevsky plane can be mapped isometrically onto suitable domains on the pseudosphere. Here the pseudosphere is, as usual, the surface in Euclidean 3-space formed by the rotation of the tractrix near its asymptote; and a domain is called special if it belongs to one of the following three classes: (i) the domains enclosed between two intersecting straight lines and a circle orthogonal to both of them, (ii) the domains enclosed between two divergent straight lines, their common perpendicular and the equidistant orthogonal to them, and (iii) the domains enclosed between two parallel straight lines and a horocycle orthogonal to both of them. However, the entire Lobachevsky plane cannot be mapped isometrically onto any domain on the pseudosphere. Therefore, the problem of finding a regular surface in Euclidean 3-space which is isometric to the entire Lobachevsky plane was of fundamental importance for 19th-century mathematics. 
However, in 1901, David Hilbert gave a negative solution to this problem, see [40]. Namely, he proved that in Euclidean 3-space there is no complete regular surface with Gauss curvature $K=$ const $<0$. As a result, the proof of the consistency of Lobachevsky geometry went a different way, while the problem of how essential is the requirement of constancy of Gauss curvature in the Hilbert's theorem remained open for a long time. Only in 1964 Nikolă V. Efimov completely removed this restriction by proving that

Theorem 1 ([24, n. 1]). No surface can be $C^{2}$-immersed in Euclidean 3-space so as to be complete in the induced Riemannian metric, with Gauss curvature $K \leqslant$ const $<0$.

In her famous article [45, p. 474], Tilla Klotz Milnor writes that Efimov's proof of Theorem 11 "is most ingenious, and does not depend upon sophisticated or modern techniques." After [24], Efimov published several more articles related to Theorem 1. Among other things, he established that the condition $K \leqslant$ const $<0$ is not the only obstacle for the immersibility of a complete surface of negative curvature. In [25], he showed that a rather slow change of Gauss curvature is another obstacle. In all those numerous articles, he used to a large extent one and the same method based on the study of the spherical image of a surface. At that study, an essential role belongs to statements that, under some conditions, locally homeomorphic mapping $f: \mathbb{R}^{2} \rightarrow \mathbb{R}^{2}$ is, in the reality, a global homeomorphism and $f\left(\mathbb{R}^{2}\right)$ is a convex domain in $\mathbb{R}^{2}$. For example, in 1968 Efimov formulated and proved the following Theorems 2 and 3 :

Theorem 2 ([26, Theorem II]). Let $f: \mathbb{R}^{2} \rightarrow \mathbb{R}^{2}$ be a continuously differentiable mapping such that det $f^{\prime}(x)<0$ for all $x \in \mathbb{R}^{2}$ and let there exist a function $a(x)>0$ and constants $C_{1} \geqslant 0, C_{2} \geqslant 0$ such that the inequalities

$$
\left|\frac{1}{a(x)}-\frac{1}{a(y)}\right| \leqslant C_{1}|x-y|+C_{2}
$$

and

$$
\left|\operatorname{det} f^{\prime}(x)\right| \geqslant a(x)|\operatorname{curl} f(x)|+a^{2}(x)
$$

hold true for all $x, y \in \mathbb{R}^{2}$. Then $f\left(\mathbb{R}^{2}\right)$ is a convex domain and $f$ maps $\mathbb{R}^{2}$ onto $f\left(\mathbb{R}^{2}\right)$ homeomorphically.

Here curl $f(x)=\partial f_{2} / \partial x_{1}(x)-\partial f_{1} / \partial x_{2}(x)$ stands for the curl of $f=\left(f_{1}, f_{2}\right)$ at $x=\left(x_{1}, x_{2}\right) \in \mathbb{R}^{2}$.

Note that if $1 / a(x)$ has exponential growth (and, thus, the condition (11) is roughly violated) then the conclusion of Theorem 2 may not hold even for potential mappings (i. e., such that curl $f(x) \equiv 0)$. For example, for $f\left(x_{1}, x_{2}\right)=\left(e^{x_{1}} \sin x_{2}, e^{x_{1}} \cos x_{2}\right)$ we have curl $f(x) \equiv 0$, det $f^{\prime}\left(x_{1}, x_{2}\right)=-e^{2 x_{1}}<0, f\left(\mathbb{R}^{2}\right)=\mathbb{R}^{2} \backslash\{(0,0)\}$ is nonconvex and every point from $f\left(\mathbb{R}^{2}\right)$ has infinite number of preimages. 
Theorem 3 ([26, Theorem 3]). Let $f: \mathbb{R}^{2} \rightarrow \mathbb{R}^{2}$ be a continuously differentiable mapping such that det $f^{\prime}(x)<0$ for all $x \in \mathbb{R}^{2}$ and let there exists $a=$ const $>0$ such that for all $x \in \mathbb{R}^{2}$ the inequality

$$
\left|\operatorname{det} f^{\prime}(x)\right| \geqslant a|\operatorname{curl} f(x)|+a^{2}
$$

holds true. Then $f\left(\mathbb{R}^{2}\right)$ is either an infinite strip between parallel straight lines, or a half-plane, or the plane, and $f$ maps $\mathbb{R}^{2}$ onto $f\left(\mathbb{R}^{2}\right)$ homeomorphically.

Note that the condition (3) of Theorem 3 is satisfied provided $\left|\operatorname{det} f^{\prime}(x)\right| \geqslant$ const $>0$ and $|\operatorname{curl} f(x)| \leqslant$ const $<+\infty$ hold true for all $x \in \mathbb{R}^{2}$.

This article is about the trace left by Theorems 2 and 3 in mathematics. More precisely, we give an overview of the analogues of these theorems, their generalizations and applications over the past 40 years. Sections 25 are devoted to presentation of results motivated by the theory of surfaces, the theory of global inverse function, the Jacobian Conjecture, and the global asymptotic stability of dynamical systems, respectively. Of course, some results can be attributed to several sections; nevertheless, we present each result only once. We also note that literature on the issues discussed in this article is extremely extensive and we are not able to cover all the results known today. The choice is limited by our personal preferences.

This article is an extended and updated version of the lecture [4 given by the author at the conference "Metric geometry of surfaces and polyhedra" dedicated to the centennial birthday anniversary of N.V. Efimov, held at Lomonosov Moscow State University in August 2010.

Nikolaŭ Vladimirovich Efimov (May 31, 1910 - August 14, 1982) was a prominent Soviet mathematician. The reader can find information about his life and scientific heritage in his obituary [1] and the book of memoirs about him [62].

\section{Results Motivated By THE THEORY OF SURFACES}

For completeness, we start with a mini-survey of results related to Theorem 1 .

A fully detailed exposition of Efimov's proof of Theorem 1 is given in the wellwritten article [45], which contributed a lot to popularity of Theorem 1 among western mathematicians. Formally speaking, Theorem 1 is related to the general problem of immersing a Riemannian metric into a Euclidean space of suitable dimension. Numerous refinements of this general problem are known; extremely diverse technologies are used to solve them, see [36], [39]. In reality, Theorem 1 has had the greatest impact on the field of mathematics known as geometry "in the large." In this field, the emphasis is on non-local properties of isometric immersions (and embeddings) of manifolds into Euclidean spaces or other spaces under various restrictions on the curvature of the immersed manifold, and on connections between geometry and topology of immersed manifolds. The reader interested in the impact of Theorem 1 on various problems of geometry "in the large" is referred to survey articles [6], [7], 14], 53], [54]. Below, we briefly mention only articles that are not included in these surveys. 
In [60], the authors study the $n$-dimensional Efimov Conjecture: if $S$ is a complete isometrically immersed hypersurface in $\mathbb{R}^{n+1}$ whose Ricci curvatures are all negative, then these curvatures are not bounded above by a negative constant. When $n=3$ the authors prove that this conjecture is correct in a very strong sense: the second fundamental form $A$ of such a hypersurface must satisfy inf $|A|=0$. In the case $n>3$, they prove a partial result: if a counterexample to the conjecture exists, then its sectional curvatures must take every real value.

In [51], the author constructs an example of a complete analytic saddle surface in $\mathbb{R}^{4}$ with Gauss curvature $K \leqslant$ const $<0$. This example shows that Theorem 1 does not generalize to saddle surfaces in $\mathbb{R}^{n}, n \geqslant 4$. Recall that a complete surface in Euclidean space is called saddle if it has no locally strictly supporting hyperplane at any point; saddle surfaces have an intrinsic metric of nonpositive curvature.

In [57], a theorem analogous to Theorem 1 is proved in the case of an ambient space of variable curvature with some extra conditions on its sectional curvature.

In [58], the author proves the following analogue of Theorem 1; Let $S$ be a complete Riemannian surface with curvature $K \leqslant$ const $<0$ such that $\|\nabla K\| \cdot|K|^{-3 / 2}$ is bounded. Then $S$ does not admit any isometric immersion neither into hyperbolic space $\mathbb{H}^{3}$ nor into spherical space $\mathbb{S}^{3}$. Similar result is also proved for Lorentz 3-space $\mathbb{H}_{1}^{3}$ of constant curvature -1 .

In [30], the authors study complete surfaces in $\mathbb{R}^{3}$ with Gauss curvature satisfying the inequality $K \leqslant$ const $<0$ in a neighborhood of infinity. They prove that such a surface is topologically a finitely punctured compact surface, its surface area is finite, and each puncture is a cusp extending to infinity, asymptotic to a ray. Similar results on complete surfaces in non-Euclidean space forms are obtained in [31.

In [47, the author studies non-compact connected surfaces $S$ with compact boundary and proves that there is no complete isometric immersion of $S$ into $\mathbb{R}^{3}$ satisfying that $\int_{S}|K|=+\infty$ and $K \leqslant$ const $<0$. In particular, he shows that Theorem [1] holds true for complete Hadamard immersed surfaces (i. e., immersed complete simply connected Riemannian manifolds with non positive sectional curvature), whose Gauss curvature $K$ is bounded away from zero outside a compact set.

In [17], [18], and [19], it is shown that Theorem 1 allows us to better understand the solutions of the Einstein-Maxwell equation of gravitation and electromagnetism in source-free space.

Summing up this mini-survey of results related to Theorem 1, we can say that it has had and continues to have a significant impact on geometry "in the large," some problems closely related to it still remain to be answered; also they attract the attention of young researchers. The latter is evidenced, for example, by the Master's Dissertation [8]. The most well-known still open problem closely related to Theorem 1 reads as follows:

Conjecture 4 (John Milnor, [45]). Suppose $S$ is a complete, umbilic free surface, $C^{2}$-immersed in $\mathbb{R}^{3}$ so that the sum of the squares of the principal curvatures on $S$ is bounded away from zero. Then either $K$ changes sign on $S$, or else $K=0$. 
Some results related to Conjecture 4 may be found in [63, 64].

Now we turn to a discussion of results which are related to Theorems 2 and 3 , were obtained in connection with the theory of surfaces, and were not presented in the survey articles [6], [7], [14], [53], [54].

Under the conditions of Theorem [3, $f\left(\mathbb{R}^{2}\right)$ is either an infinite strip between parallel straight lines, or a half-plane, or the plane. Results clarifying which of these possibilities may actually occur can be found in the articles [43] and [32], book [10, $\S 31$ ], and Ph. D. thesis [52]. Let us describe them in some detail.

The case, when $f\left(\mathbb{R}^{2}\right)$ is the plane, is, obviously, realized by the linear mapping $f_{1}\left(x_{1}, x_{2}\right)=x_{2}, f_{2}\left(x_{1}, x_{2}\right)=x_{1}$.

The case, when $f\left(\mathbb{R}^{2}\right)$ is a half-plane, is realized by the mapping $f: \mathbb{R}^{2} \rightarrow \mathbb{R}^{2}$ defined by the formulas found in [43]:

$$
f_{1}\left(x_{1}, x_{2}\right)=\log \left(x_{1}+\sqrt{x_{1}^{2}+e^{-2 x_{2}}}\right)+x_{2}, \quad f_{2}\left(x_{1}, x_{2}\right)=\sqrt{x_{1}^{2}+e^{-2 x_{2}}} .
$$

In fact, it is easy to check directly that for this mapping we have

$$
\operatorname{det} f^{\prime}(x)=\text { const }<0, \quad \operatorname{curl} f(x)=0,
$$

and $f\left(\mathbb{R}^{2}\right)$ is the half-plane $f_{2}>0$. The formulas (4) look complicated, but they can be obtained in the following natural way proposed in [43. Note that the conditions (5) will certainly be satisfied if $f=\left(f_{1}, f_{2}\right)$ is potential (i. e., if there exists a function $\varphi: \mathbb{R}^{2} \rightarrow \mathbb{R}$, called a potential, such that $f_{j}=\partial \varphi / \partial x_{j}$ for $\left.j=1,2\right)$ and the potential $\varphi$ satisfies the Monge-Ampère equation

$$
\frac{\partial^{2} \varphi}{\partial x_{1}^{2}} \frac{\partial^{2} \varphi}{\partial x_{2}^{2}}-\left(\frac{\partial^{2} \varphi}{\partial x_{1} \partial x_{2}}\right)^{2}=-1
$$

Then using the classical parametric representation for solutions of the MongeAmpère equation [35, Section 476, example 4], we obtain a large class of non-trivial examples of mappings $f: \mathbb{R}^{2} \rightarrow \mathbb{R}^{2}$ satisfying the conditions (5) for which $f\left(\mathbb{R}^{2}\right)$ is a half-plane. The formulas (4) define just one mapping from that class.

The case, when $f\left(\mathbb{R}^{2}\right)$ is an infinite strip between parallel straight lines was studied in [43] and [32]. More precisely, in [43] it is proved that this case is impossible provided that the conditions (5) are fulfilled. Moreover, in [43] it is conjectured that this case can never occur under the conditions of Theorem 3 . This conjecture remains open to this day. Partial results on this conjecture are obtained in [32], where it is shown that $f\left(\mathbb{R}^{2}\right)$ cannot be a strip provided that curl $f(x)=0$ and $\operatorname{det} f^{\prime}(x)=-g^{2}(x)$ with some function $g$, which satisfies the inequality $g(x) \geqslant$ const $>0$ and is either a convex function or a polynomial.

We proceed by describing the results obtained by G. Ya. Perel'man in his Ph. D. dissertation [52]. The dissertation is devoted to the study of saddle surfaces in $\mathbb{R}^{n}$, $n \geqslant 3$, i. e., to generalization and strengthening of theorems proved by Efimov in [24], [25], 26] and, in particular, of Theorem 1. Among other results, G. Ya. Perel'man proved the following theorem which, as far as we know, was never published in a peer reviewed journal: 
Theorem 5 ([52, p. 78]). Let $f: \mathbb{R}^{2} \rightarrow \mathbb{R}^{2}$ be a potential orientation-reversing global diffeomorphism of class $C^{1}$. Then $f\left(\mathbb{R}^{2}\right)$ is a domain from the following list: (1) a triangle; (2) a quadrangle; (3) the plane; (4) a half-plane; (5) a strip between two parallel lines; (6) an angle; (7) a half-strip; (8) an angle with a cut off vertex; (9) a half-strip with a cut off vertex.

Note that the conditions of Theorem 5 do not control the decay of $\operatorname{det} f^{\prime}(x)$ at infinity at all, i.e., no condition similar to the inequalities (2) or (3) is imposed. The proof of Theorem 5 is a clever modification of the Efimov's method, proposed in [24], [25], [26].

We complete this Section with a description of an extension of Theorem 1 to surfaces in the three-dimensional Heisenberg group Nil published in [13. Recall that the Heisenberg group $N i l$ is $\mathbb{R}^{3}$ endowed with the metric

$$
d s^{2}=d x^{2}+d y^{2}+\left(\frac{1}{2} y d x-\frac{1}{2} x d y+d z\right)^{2} .
$$

Here $x, y, z$ are global Cartesian coordinates in $\mathbb{R}^{3}$. It is known that, in the Heisenberg group, surfaces with Gauss curvature bounded above by a negative constant do exist. However, the following analogue of Theorem 1 holds true for surfaces, which are explicitly given in coordinates $(x, y)$ :

Theorem 6 ([13, Theorem 3]). In Nil, on any $C^{2}$-surface $z=f(x, y)$ explicitly given in the entire plane $(x, y)$, the greatest lower bound of the absolute value of the Jacobian of Gauss mapping is zero.

In Theorem 6, the replacement of Gauss curvature by the Jacobian of Gauss mapping looks quiet natural, and Gauss mapping of a surface in Nil is defined as Gauss mapping of a surface in a Lie group. For our exposition it is also important that Theorem 3 is used essentially in the proof of Theorem 6. Indeed, the key point of the proof of Theorem [6 is that the mapping $F: \mathbb{R}^{2} \rightarrow \mathbb{R}^{2}$ defined as the composition of Gauss mapping and the projection of the sphere from the origin onto its tangent plane at the point $(0,0,1)$ satisfies the conditions of Theorem 3 and, thus, is a homeomorphism of $\mathbb{R}^{2}$ onto a convex domain $F\left(\mathbb{R}^{2}\right)$ in $\mathbb{R}^{2}$.

\section{Results motivated By THEOREMS ON GLOBAL INVERSE FUNCTION}

Theorems on global inverse function play an important role in many fields of Analysis and its applications, including mathematical economics. The interested reader may find a broad range of such theorems, e.g., in [50], [49, [36], and [41. Geometric applications may be found, e.g., in the book [56] devoted to the study of isometric immersions and embeddings of locally Euclidean metrics using various theorems on the univalence of mappings, and in the survey paper [12 devoted to the study of univalent solutions of the uniformly elliptic Beltrami equation realizing a homeomorphic quasiconformal mapping of the complex plane with the assigned measurable complex dilatation. 
To the best of our knowledge, the first result on existence of a global inverse function was obtained by Jacques Hadamard in [38] for mappings $\mathbb{R}^{2} \rightarrow \mathbb{R}^{2}$. Nowadays the following statement of the global inverse function theorem is considered standard:

Theorem 7 ([50, Theorem 3.2]). Let $X$ and $Y$ be Banach spaces, $f: X \rightarrow Y \in$ $C^{1}(X)$ and $f^{\prime}(x)$ is invertible for all $x \in X$. If

$$
\int_{0}^{+\infty} \inf _{\|x\| \leqslant t}\left\|f^{\prime}(x)^{-1}\right\|^{-1} d t=+\infty
$$

then $f$ is a diffeomorphism of $X$ onto $Y$.

Note that the condition ([6] of Theorem 7 controls the growth of the norm $\left\|f^{\prime}(x)^{-1}\right\|$ at infinity and is obviously fulfilled in the case, when

$$
\left\|f^{\prime}(x)^{-1}\right\| \leqslant A=\text { const }<+\infty
$$

for all $x \in X$.

Hadamard's theorem and its modern versions, e. g., Theorem [7, admit numerous generalizations to nonsmooth mappings. As an example, we mension the following theorem

Theorem 8 ([9, Theorem 2.2]). Let $n \geqslant 1, \alpha>0$, and let $f: \mathbb{R}^{n} \rightarrow \mathbb{R}^{n}$ be continuous, locally injective and $\alpha$-covering at each $x \in \mathbb{R}^{n}$ (the latter means that, for every $\varepsilon>0$, there exists $r \in(0, \varepsilon]$ such that $B(f(x), \alpha r) \subset f(B(x, r))$, where $B(x, r)$ stands for the ball of radius $r$ centered at $x)$. Then $f$ is a global homeomorphism and $f^{-1}$ is Lipschitz continuous with the constant $\alpha^{-1}$.

In [2], it is proved that the following theorem is equivalent to Theorem 2,

Theorem 9 ([2, Theorem 4]). Let $f: \mathbb{R}^{2} \rightarrow \mathbb{R}^{2}$ be a continuously differentiable mapping such that $\operatorname{det} f^{\prime}(x)<0$ for all $x \in \mathbb{R}^{2}$; and let there exist a function $a(x)>0$ and constants $C_{1} \geqslant 0, C_{2} \geqslant 0$ such that the inequality (1) holds true for all $x, y \in \mathbb{R}^{2}$ and, for all $x \in \mathbb{R}^{2}$, the inequality

$$
\left|\mu_{2}(x)\right| \geqslant\left|\mu_{1}(x)\right| \geqslant a(x)
$$

holds true. Here $\mu_{1}(x)$ and $\mu_{2}(x)$ are the eigenvalues of $f^{\prime}(x)$. Then $f$ maps $\mathbb{R}^{2}$ onto $f\left(\mathbb{R}^{2}\right)$ homeomorphically, and $f\left(\mathbb{R}^{2}\right)$ is convex.

Note that the inequalities (8) control the decrease of the spectral radius $\rho\left(f^{\prime}(x)\right)$ of $f^{\prime}(x)$ at infinity and are obviously fulfilled in the case, when

$$
\left|\mu_{2}(x)\right| \geqslant\left|\mu_{1}(x)\right| \geqslant a=\text { const }>0
$$

for all $x \in \mathbb{R}^{2}$. As usual, the spectral radius of a linear mapping is equal to the maximum of the modules of its eigenvalues. In other words, we can say that the 
inequalities (8) control the growth of the spectral radius $\rho\left(f^{\prime}(x)^{-1}\right)$ of $f^{\prime}(x)^{-1}$ at infinity and are obviously fulfilled in the case, when

$$
\rho\left(f^{\prime}(x)^{-1}\right) \leqslant A=1 / a=\text { const }<+\infty
$$

for all $x \in \mathbb{R}^{2}$. Comparing (7) and (9), we arrive at the following conjecture:

Conjecture $10\left(\left[2\right.\right.$, p. 199], [3, p. 104]). Let $n \geqslant 2, f: \mathbb{R}^{n} \rightarrow \mathbb{R}^{n}$ be a continuously differentiable mapping such that $\operatorname{det} f^{\prime}(x) \neq 0$ and

$$
\rho\left(f^{\prime}(x)^{-1}\right) \leqslant A
$$

for all $x \in \mathbb{R}^{n}$ with some constant $A<+\infty$. Then $f\left(\mathbb{R}^{n}\right)$ is a convex domain in $\mathbb{R}^{n}$ and $f$ is injective.

Note that Theorem 9 gives the affirmative answer to Conjecture 10 in the case, when $n=2$ and $\operatorname{det} f^{\prime}(x)<0$. Note also that Conjecture 10 is in agreement with Theorem 7 in the following sense. Let $n \geqslant 2, f: \mathbb{R}^{n} \rightarrow \mathbb{R}^{n}$ be a continuously differentiable mapping, $f^{\prime}(x)$ be invertible and $\left\|f^{\prime}(x)^{-1}\right\| \leqslant A=$ const $<+\infty$ for all $x \in \mathbb{R}^{n}$. Then, according to Theorem $7, f$ is a diffeomorphism of $\mathbb{R}^{n}$ onto $\mathbb{R}^{n}$. At the same time, we see that such $f$ satisfies both the conditions of Conjecture 10 (since $\rho\left(f^{\prime}(x)^{-1}\right) \leqslant\left\|f^{\prime}(x)^{-1}\right\| \leqslant A$ ) and its conclusion (since $f$ is injective and $f\left(\mathbb{R}^{n}\right)=\mathbb{R}^{n}$ is convex .

Conjecture 10 is apparently very natural and was formulated by different researchers in connection with problems from various fields of mathematics. For example, in the article [2] and conference proceedings [3] it was formulated in the form given above and was motivated by its connection with Efimov's Theorem 2 , In [16, Conjecture 2.1], it was formulated without mentioning that $f\left(\mathbb{R}^{n}\right)$ is convex and was motivated by its connection with the Jacobian Conjecture of Ott-Heinrich Keller [44]; see Section 4 below for more details. Only during the preparation of this survey article, we found that in fact for the first time Conjecture 10 was formulated in [46] in connection with Efimov's Theorem 2. Moreover, the following theorem is proved there:

Theorem 11 ([46, p. 224]). Let $f: \mathbb{R}^{2} \rightarrow \mathbb{R}^{2}$ be a continuously differentiable mapping such that the eigenvalues $\mu_{1}(x), \mu_{2}(x)$ of $f^{\prime}(x)$ satisfy everywhere the conditions:

(1) $\mu_{1}(x)$ and $\mu_{2}(x)$ are real;

(2) $\mu_{1}(x) \neq \mu_{2}(x)$;

(3) $0<a \leqslant \min \left\{\left|\mu_{1}(x)\right|,\left|\mu_{2}(x)\right|\right\}$ with some $a=$ const.

Then $f$ is injective and $f\left(\mathbb{R}^{2}\right)$ is convex.

Note that Theorem 11 is applicable to both orientation-reversing and orientationpreserving mappings $f$. The proof of Theorem 11 follows closely Efimov's proof of his Theorem 2 ,

To conclude this Section, we point out another natural problem related to Conjecture 10, which remains open so far. It reads as follows: find an estimation of 
the radius of the ball, where the inverse mapping $f^{-1}$ exists, in terms of the spectral radius $\rho\left(f^{\prime}(x)^{-1}\right)$. The estimation is expected to be similar to that in the following theorem:

Theorem 12 ([42, Theorem IIA]). Let $X$ and $Y$ be Banach spaces, $B$ be the ball of center $x_{0}$ and radius $r$ in $X$, and $f: B \rightarrow Y$ be a continuously differentiable mapping, whose derivative $f^{\prime}(x)$ is invertible for all $x \in B$. Then the inverse mapping $f^{-1}$ exists in the ball of center $f\left(x_{0}\right)$ and radius

$$
R=\int_{0}^{r} \inf _{\left\|x-x_{0}\right\| \leqslant t}\left\|f^{\prime}(x)^{-1}\right\|^{-1} d t .
$$

Theorem 12 generalizes Theorem 7 and is quoted here in our notation.

\section{Results motivated By the Jacobian Conjecture}

A mapping $f: \mathbb{C}^{n} \rightarrow \mathbb{C}^{n}$ is said to be polynomial if $f=\left(f_{1}, \ldots, f_{n}\right)$ and each component $f_{i}$ of $f$ is a polynomial. A polynomial mapping $f$ is said to be a polynomial automorphism of $\mathbb{C}^{n}$ if $f$ is one-to-one, $f\left(\mathbb{C}^{n}\right)=\mathbb{C}^{n}$, and $f^{-1}: \mathbb{C}^{n} \rightarrow \mathbb{C}^{n}$ is also a polynomial mapping.

Conjecture 13 (Jacobian Conjecture, [44]). For every positive integer $n$ and every polynomial mapping $f: \mathbb{C}^{n} \rightarrow \mathbb{C}^{n}$, whose Jacobian is a non-zero constant, $f$ is a polynomial automorphism of $\mathbb{C}^{n}$.

The Jacobian Conjecture was first formulated by Ott-Heinrich Keller in 1939 but is still unproved, even for $n=2$. Its history, many references, and some partial results can be found in [65]. Stephen Smale included the Jacobian Conjecture in his list of eighteen problems for the 21st century [59, Problem 16]. Note that various additional conditions on $f$ are known under which the Jacobian Conjecture is certainly valid. One of such additional conditions is the requirement that $f$ is injective. An elementary proof of this fact is given in [55]. This explains the interest of specialists on the Jacobian Conjecture in conditions guaranteeing the injectivity of mappings. We indicate one of their conjectures in our notation:

Conjecture 14 (M. Chamberland, [16, Conjecture 2.1]). Let $n \geqslant 2, f: \mathbb{R}^{n} \rightarrow \mathbb{R}^{n}$ be a continuously differentiable mapping such that $\operatorname{det} f^{\prime}(x) \neq 0$ and

$$
\rho\left(f^{\prime}(x)^{-1}\right) \leqslant A
$$

for all $x \in \mathbb{R}^{n}$ with some constant $A<+\infty$, where $\rho\left(f^{\prime}(x)^{-1}\right)$ is the spectral radius of $f^{\prime}(x)^{-1}$. Then $f$ is injective.

Note that the condition (10) can be replaced (and is replaced in [16]) by the equivalent requirement that there exists an $\varepsilon>0$ such that $|\lambda| \geqslant \varepsilon$ for all the eigenvalues $\lambda$ of $f^{\prime}(x)$ for all $x \in \mathbb{R}^{n}$. Note also that, strictly speaking, Conjecture 14 is weaker than Conjecture 10, since it does not state that $f\left(\mathbb{R}^{n}\right)$ is convex. However, 
Conjecture 14 also remains open. We consider the following theorem as closely related to Conjecture 14:

Theorem 15 ([16, Theorem 1.1]). Let $n \geqslant 2$ and $f: \mathbb{R}^{n} \rightarrow \mathbb{R}^{n}$ be a continuously differentiable mapping. Suppose there exists an $\varepsilon>0$ such that $|\mu| \geqslant \varepsilon$ for all the eigenvalues $\mu$ of $f^{\prime}(x) f^{\prime}(x)^{T}$ for all $x \in \mathbb{R}^{n}$. Then $f$ is injective.

To prove Theorem 15, the authors use the so-called Mountain Pass Lemma due to [5], i. e., they use techniques which differs very much from those used by Efimov to prove Theorems 2 and 3 .

\section{Results motivated By THE GLOBAL ASYMPTOTIC STABILITy OF DYNAMICAL SYSTEMS}

Conjectures similar to Conjectures 10 and 14 appeared also in the study of the global asymptotic stability of dynamical systems in the plane. Namely, in this field of mathematics several authors conjectured that some restrictions on the set of all eigenvalues of $f^{\prime}(x)$ (like separation from zero) already imply the injectivity of a $C^{1}$ mapping $f: \mathbb{R}^{n} \rightarrow \mathbb{R}^{n}$. In this Section we present some conjectures and theorems of this sort.

The problem of the global asymptotic stability of dynamical systems in the plane can be formulated as follows: Consider an autonomous system in the plane

$$
\left\{\begin{array}{l}
\dot{x}_{1}=f_{1}\left(x_{1}, x_{2}\right) \\
\dot{x}_{2}=f_{2}\left(x_{1}, x_{2}\right)
\end{array}\right.
$$

for which $\left(x_{1}, x_{2}\right)=(0,0)$ is an equilibrium point $\left(i . e ., f_{1}(0,0)=f_{2}(0,0)=0\right)$ and the eigenvalues of the Jacobi matrix $f^{\prime}(x)$ of the mapping $f=\left(f_{1}, f_{2}\right)$ have negative real parts at any point $x$ on the plane. Is it then true that the trivial solution $\left(x_{1}, x_{2}\right)=(0,0)$ is globally asymptotically stable, $i . e$. , that any solution of the equations (11) tends to the point $(0,0)$ as $t \rightarrow \infty$ ? This problem plays an important role in the theory and applications of dynamical systems, see, e.g., [21]. According to the famous Lyapunov theorem, an autonomous system of linear differential equations $\dot{y}=A y, y \in \mathbb{R}^{n}, n \geqslant 2$, is globally asymptotically stable if all the eigenvalues of the constant matrix $A$ have negative real parts. Thus, the problem of the global asymptotic stability answers the question of whether it is possible to make a conclusion about the global asymptotic stability of any solution of the nonlinear system (11) as soon as the global asymptotic stability of any solution of the linearized system $\dot{y}=f^{\prime}(x) y$ is established for all $x \in \mathbb{R}^{2}$.

The problem of the global asymptotic stability of dynamical systems can be posed in $\mathbb{R}^{n}$ for arbitrary $n$. It is known as the Markus-Yamabe Conjecture and is known to be false for $n \geqslant 3$, see, e.g., [22].

In [48, a close connection is discovered between the problem of the global asymptotic stability in the plane and injectivity of some mappings; namely, the following theorem is proved there: 
Theorem 16 ([48]). The following two statements are equivalent to each other: (1) the problem of the global asymptotic stability in the plane has the affirmative answer; (2) every continuously differentiable mapping $f: \mathbb{R}^{2} \rightarrow \mathbb{R}^{2}$, the eigenvalues of the Jacobi matrix $f^{\prime}$ of which everywhere have negative real parts, is injective.

In 1994-1995, the affirmative answer to the problem of the global asymptotic stability in the plane was obtained in [33], [34], [29], and [37]. The proofs proposed in these articles rely on Theorem 16. However, interest in the problem of the global asymptotic stability in the plane does not disappear; on the contrary, more and more new proofs appear, see, e.g., [20]. Moreover, specialists in different fields of mathematics invent and study various differential tests for injectivity of mappings related to Theorems 2 and 3, and Conjectures [10 and 14. We consider the following theorem as a very important contribution to this field of research:

Theorem $17\left(\left[23\right.\right.$, Theorem A]). Let $f: \mathbb{R}^{2} \rightarrow \mathbb{R}^{2}$ be a continuously differentiable mapping, and let $\operatorname{Spec}(f)$ be the set of all eigenvalues of the Jacobian matrix $f^{\prime}(x)$ for all $x \in \mathbb{R}^{2}$. Suppose that $\operatorname{Spec}(f) \cap(-\varepsilon, \varepsilon)=\varnothing$ for some $\varepsilon>0$. Then $f$ is injective.

The key point in the proof of Theorem 17 is the notion of a half-Reeb component of a planar vector field. In particular, the authors use techniques which is very different from those used by Efimov to prove Theorems 2 and 3. Note that, for $n=2$, Theorem 17 shows that a statement even stronger than Conjecture 14 holds true. Namely, in this case for injectivity of $f$ it is sufficient to require that $\operatorname{Spec}(f)$ does not intersect with a sufficiently small interval $(-\varepsilon, \varepsilon)$ of the real axis $\mathbb{R}$ only, not with the entire disk of radius $\varepsilon$ centered at 0 in the complex plane $\mathbb{C}$. In [27], it is shown that, in Theorem 17, the condition $\operatorname{Spec}(f) \cap(-\varepsilon, \varepsilon)=\varnothing$ can be replaced by the weaker condition $\operatorname{Spec}(f) \cap[0, \varepsilon)=\varnothing$. On the other hand in [61], it is proved that there exist integers $n>2$ and non-injective polynomial mappings $f: \mathbb{R}^{n} \rightarrow \mathbb{R}^{n}$ with $\operatorname{Spec}(f) \cap[0,+\infty)=\varnothing$. Nevertheless, some partial extensions of Theorem 17 to $\mathbb{R}^{n}, n>2$, are obtained in [61] and [28].

At last, we would like to mention the article [15], where the author explores whether the $C^{1}$-mappings with a unipotent (i.e., all eigenvalues are 1) Jacobian matrix are injective. He extends some results known in the polynomial case to the $C^{1}$ context, resolves some special cases, and discusses connections between the problem of the injectivity of the $C^{1}$-mappings with a unipotent Jacobian matrix and the Jacobian Conjecture 13, Chamberland's Conjecture 14, and a few additional ones that have arisen in connection with the study of the Markus-Yamabe Conjecture and some its discrete analogue. One of conjectures discussed in [15] reads as follows:

Conjecture $18\left(C^{1}\right.$ Fixed Point Conjecture, [15, Conjecture 5]). If $f: \mathbb{R}^{n} \rightarrow \mathbb{R}^{n}$ is $C^{1}$ with $f(0)=0$, and the eigenvalues of $f^{\prime}(x)$ have absolute value less than 1 at every point $x \in \mathbb{R}^{n}$, then 0 is the unique fixed point of $f$.

According to [15, being applied to polynomial mappings Conjecture 18 is equivalent to the Jacobian Conjecture 13 , 


\section{Conclusion}

Theorems deducing the injectivity of mappings $f: \mathbb{R}^{n} \rightarrow \mathbb{R}^{n}$ from some properties of $f^{\prime}(x)$ appear in different branches of mathematics. Efimov's Theorems 2 and 3 are among the first results of this type. They directly stimulated the studies of some mathematicians, or later turned out to be closely related to findings of other mathematicians not familiar with the works of Efimov. Open problems related to Theorems 2 and 3 continue to attract the attention of mathematicians.

\section{REFERENCES}

[1] Aleksandrov, A.D., Novikov, S.P., Pogorelov, A.V., Poznyak, É.G., Rashevskiŭ, P.K., Rozendorn, É.R., Sabitov, I.Kh., Stechkin, S.B.: Nikolaŭ Vladimirovich Efimov (obituary). Russ. Math. Surv. 38(5), 123-130 (1983)

[2] Aleksandrov, V.A.: On Efimov's theorem on differential tests for a homeomorphism. Math. USSR, Sb. 69(1), 197-202 (1990)

[3] Alexandrov, V.A.: Remarks on Efimov's theorem about differential tests of homeomorphism. Rev. Roum. Math. Pures Appl. 36(3-4), 101-105 (1991)

[4] Alexandrov, V.A.: On a differential test of homeomorphism, found by N.V. Efimov. (In Russian). Contemp. Probl. Math. Mech. 6(2), 18-26 (2011)

[5] Ambrosetti, A., Rabinowitz, P.H.: Dual variational methods in critical point theory and applications. J. Funct. Anal. 14, 349-381 (1973)

[6] Aminov, Yu.A.: Embedding problems: Geometric and topological aspects. J. Sov. Math. 25, 1308-1331 (1984)

[7] Aminov, Yu.A.: Two-dimensional surfaces in 3-dimensional and 4-dimensional Euclidean spaces. Results and unsolved problems. Ukr. Math. J. 71(1), 1-38 (2019)

[8] Araújo, Laís Pinheiro da Silva: O problema de Hilbert - Cohn-Vossen nos espaços tridimensionais. Dissertacão de Mestrado; pp. 1-62. Brasília: Universidade de Brasília (2019)

[9] Arutyunov, A.V., Zhukovskiy, S.E.: Hadamard's theorem for mappings with relaxed smoothness conditions. Sb. Math. 210(2), 165-183 (2019)

[10] Bakel'man, I.Ya., Verner, A.L., Kantor, B.E.: Introduction to differential geometry "in the large". (In Russian). Moscow: Nauka (1973)

[11] Beltrami, E.: Saggio di interpretazione della Geometria non-euclidea. Battaglini G. 6, 285-315 (1868)

[12] Bojarski, B.: On the Beltrami equation, once again: 54 years later. Ann. Acad. Sci. Fenn., Math. 35(1), 59-73 (2010)

[13] Borisenko, A.A., Petrov, E.V.: Surfaces in the three-dimensional Heisenberg group on which the Gauss map has bounded Jacobian. Math. Notes 89(5), 746-748 (2011)

[14] Burago, Yu.D., Shefel', S.Z.: The geometry of surfaces in Euclidean spaces. In: Burago, Yu.D. (ed.) et al., Geometry III. Theory of surfaces. Encycl. Math. Sci. Vol. 48, 1-85. Berlin: SpringerVerlag (1992)

[15] Campbell, L.A.: Unipotent Jacobian matrices and univalent maps. In: Chan, Kai Yuen (ed.) et al., Combinatorial and computational algebra. International conference on combinatorial and computational algebra, May 24-29, 1999, Hong Kong, China, pp. 157-177. Providence: American Mathematical Society (2000)

[16] Chamberland, M., Meisters, G.: A mountain pass to the Jacobian conjecture. Can. Math. Bull. 41(4), 442-451 (1998)

[17] Chan, H.: Nonexistence of nonpositively curved surfaces with one embedded end. Manuscr. Math. 102(2), 177-186 (2000) 
[18] Chan, H.: Embedding Misner and Brill-Lindquist initial data for black-hole collisions. Math. Phys. Anal. Geom. 6(1), 9-27 (2003)

[19] Chan, H.: Embedding negatively curved initial data of black-hole collisions in $\mathbb{R}^{3}$. Classical Quantum Gravity 23(1), 225-234 (2006)

[20] Chen, P.N., He, J.X., Qin, H.S.: A proof of the Jacobian conjecture on global asymptotic stability. Acta Math. Sin., Engl. Ser. 17(1), 119-132 (2001)

[21] Chicone, C.: Ordinary differential equations with applications. 2nd ed. Texts Appl. Math. Vol. 34. New York: Springer (2006)

[22] Cima, A., van den Essen, A., Gasull, A., Hubbers, E., Mañosas, F.: A polynomial counterexample to the Markus-Yamabe conjecture. Adv. Math. 131(2), 453-457 (1997)

[23] Cobo, M., Gutierrez, C., Llibre, J.: On the injectivity of $C^{1}$ maps of the real plane. Can. J. Math. 54(6), 1187-1201 (2002)

[24] Efimov, N.V.: The appearance of singularities on surfaces of negative curvature. (In Russian). Mat. Sb., Nov. Ser. 64, 286-320 (1964)

[25] Efimov, N.V.: Surfaces with a slowly changing negative curvature. Russ. Math. Surv. 21(5), $1-55(1966)$

[26] Efimov, N.V.: Differential criteria for homeomorphism of certain mappings with applications to the theory of surfaces. Math. USSR, Sb. 5, 475-488 (1968)

[27] Fernandes, A., Gutierrez, C., Rabanal, R.: Global asymptotic stability for differentiable vector fields of $\mathbb{R}^{2}$. J. Differ. Equations 206(2), 470-482 (2004)

[28] Fernandes, A., Gutierrez, C., Rabanal, R.: On local diffeomorphisms of $\mathbb{R}^{n}$ that are injective. Qual. Theory Dyn. Syst. 4(2), 255-262 (2004)

[29] Feßler, R.: A proof of the two-dimensional Markus-Yamabe stability conjecture and a generalization. Ann. Pol. Math. 62(1), 45-74 (1995)

[30] Gálvez, J.A., Martínez, A., Teruel, J.L.: Complete surfaces with ends of non positive curvature. Adv. Math. 281, 1202-1215 (2015)

[31] Gálvez, J.A., Martínez, A., Teruel, J.L.: Complete surfaces with non-positive extrinsic curvature in $\mathbb{H}^{3}$ and $\mathbb{S}^{3}$. J. Math. Anal. Appl. 430(2), 1058-1064 (2015)

[32] Geǔsberg, S.P.: On properties of the normal mapping generated by the equation $r t-s^{2}=$ $-f^{2}(x, y)$. Mat. Sb., Nov. Ser. 82, 224-232 (1970)

[33] Glutsyuk, A.A.: A complete solution of the Jacobian problem for vector fields on the plane. Russ. Math. Surv. 49(3), 185-186 (1994)

[34] Glutsyuk, A.A.: Asymptotic stability of linearizations of a planar vector field with a singular point implies global stability. Funct. Anal. Appl. 29(4), 238-247 (1995)

[35] Goursat, É.: A course in mathematical analysis. Vol. III, Part 1: Variation of solutions. Partial differential equations of the second order. Vol. III, Part 2: Integral equations. Calculus of variations. New York: Dover Publications (1964)

[36] Gromov, M.: Geometric, algebraic, and analytic descendants of Nash isometric embedding theorems. Bull. Am. Math. Soc., New Ser. 54(2), 173-245 (2017)

[37] Gutierrez, C.: A solution to the bidimensional global asymptotic stability conjecture. Ann. Inst. Henri Poincaré, Anal. Non Linéaire 12(6), 627-671 (1995)

[38] Hadamard, J.: Sur les transformations ponctuelles. Bull. Soc. Math. Fr. 34, 71-84 (1906)

[39] Han, Q., Hong, J.X.: Isometric embedding of Riemannian manifolds in Euclidean spaces., vol. 130. Providence: American Mathematical Society (2006)

[40] Hilbert, D.: Über Flächen von konstanter Gaußscher Krümmung. Trans. Am. Math. Soc. 2, 87-99 (1901)

[41] Jaramillo, J.A., Lajara, S., Madiedo, O.: Inversion of nonsmooth maps between Banach spaces. Set-Valued Var. Anal. 27(4), 921-947 (2019)

[42] John, F.: On quasi-isometric mappings. I. Commun. Pure Appl. Math. 21, 77-110 (1968) 
[43] Kantor, B.E.: On the problem of the normal image of a complete surface of negative curvature. Math. USSR, Sb. 11, 197-200 (1970)

[44] Keller, O.-H.: Ganze Cremona-Transformationen. Monatsh. Math. Phys. 47, 299-306 (1939)

[45] Klotz Milnor, T.: Efimov's theorem about complete immersed surfaces of negative curvature. Adv. Math. 8, 474-543 (1972)

[46] Koutroufiotis, D.: Univalent mappings of the plane into itself. Bull. Greek Math. Soc. 18, 224-236 (1977)

[47] Mendonça, S.: Complete negatively curved immersed ends in $\mathbb{R}^{3}$. Geom. Dedicata 195, 319337 (2018)

[48] Olech, C.: On the global stability of an autonomous system on the plane. Contrib. Differ. Equations. Vol. 1, 389-400 (1963)

[49] Parthasarathy, T.: On global univalence theorems. Lect. Notes Math. Vol. 977. Berlin: Springer-Verlag (1983)

[50] Plastock, R.: Homeomorphisms between Banach spaces. Trans. Am. Math. Soc. 200, 169-183 (1974)

[51] Perel'man, G.Ya.: Example of a complete saddle surface in $\mathbb{R}^{4}$ with Gaussian curvature bounded away from zero. J. Sov. Math. 59(2), 760-762 (1989)

[52] Perel'man, G.Ya.: Saddle surfaces in Euclidean spaces. (In Russian). Ph.D. Thesis; pp. 186. Leningrad: Leningrad Department of Steklov Institute of Mathematics of the Academy of Sciences of the USSR (1990)

[53] Rozendorn, É.R.: Surfaces of negative curvature. In: Burago, Yu.D. (ed.) et al., Geometry III. Theory of surfaces. Encycl. Math. Sci. Vol. 48, 87-178. Berlin: Springer-Verlag (1992)

[54] Rozendorn, É.R., Shikin, E.V.: N.V. Efimov's works on the surfaces with negative curvature. (In Russian). Contemp. Probl. Math. Mech. 6(2), 49-56 (2011)

[55] Rudin, W.: Injective polynomial maps are automorphisms. Am. Math. Mon. 102(6), 540-543 (1995)

[56] Sabitov, I.Kh.: Isometric immersions and embeddings of locally Euclidean metrics. Rev. Math. Math. Phys. 13(1), 1-286 (2008)

[57] Schlenker, J.-M.: Un analogue du théorème d'Efimov en courbure variable. In: Séminaire de théorie spectrale et géométrie. Année 1994-1995, pp. 67-79. St. Martin d'Hères: Univ. de Grenoble I, Institut Fourier (1995)

[58] Schlenker, J.-M.: Surfaces à courbure extrinsèque négative dans l'espace hyperbolique. Ann. Sci. Éc. Norm. Supér. (4) 34(1), 79-130 (2001)

[59] Smale, S.: Mathematical problems for the next century. In: Arnold, V. (ed.) et al., Mathematics: frontiers and perspectives, pp. 271-294. Providence: American Mathematical Society (2000)

[60] Smyth, B., Xavier, F.: Efimov's theorem in dimension greater than two. Invent. Math. 90, 443-450 (1987)

[61] Smyth, B., Xavier, F.: Injectivity of local diffeomorphisms from nearly spectral conditions. J. Differ. Equations 130(2), 406-414 (1996)

[62] Tikhomirov, V.M., Sabitov, I.Kh. (eds.): Remembering Nikolaŭ Vladimirovich Efimov ... (In

Russian). Moscow: Moscow Center for Continuous Mathematical Education (2014)

[63] Toponogov, V.A.: On conditions for existence of umbilical points on a convex surface. Sib. Math. J. 36(4), 780-786 (1995)

[64] Toponogov, V.A.: A uniqueness theorem for convex surfaces with no umbilical points and interrelated principal curvatures. Sib. Math. J. 37(5), 1037-1040 (1996)

[65] van den Essen, A.: Polynomial automorphisms and the Jacobian conjecture. Prog. Math. Vol. 190. Basel: Birkhäuser (2000) 
Sobolev Institute of Mathematics, Koptyug ave., 4, Novosibirsk, 630090, Russia and Department of Physics, Novosibirsk State University, Pirogov str., 2, NovosiBIRSK, 630090, RUSSIA

E-mail address: alex@math.nsc.ru 